\title{
Modified Constraint Induced Movement Therapy Combined with Anticipatory Postural Adjustments Leads to Improved Corticospinal Tract in Hemiplegic Patients
}

\author{
In-Tae Ryu, PT, PhD ${ }^{1}$, Hyo-Jung Park, PT, MS ${ }^{1}$, Young-Dong Kim, PT, PhD ${ }^{* 2}$ \\ ${ }^{1}$ Dept. of Physical Therapy, Daejeon Rehabilitation Hospital, Republic of Korea \\ ${ }^{* 2}$ Dept. of Physical Therapy, Reddy Care Physical \& Occupational Therapy, USA
}

\begin{abstract}
Purpose This study aimed to verify whether modified constraint induced movement therapy combined with anticipatory postural adjustments improve corticospinal tract in hemiplegic patients. Methods Nine hemiplegic patients after stroke performed a shaping exercise which consists of Modified Constraint Induced Movement therapy (mCIMT) combined with Anticipatory Postural Adjustments (APAs) for 30 minutes per day, 5 days a week, for 4 weeks. And, the subjects were tested with Motor Activity Log (MAL) and Diffusion Tensor Imaging (DTI) before and after the intervention. Results The amount of use in MAL (MAL AOU, and Unit, core) increased from $32.00 \pm 32.04$ to $65.25 \pm 23.63$ with a significant difference. The quality of movement in MAL (MAL QOM, and Unit, score) increased from $55.63 \pm 21.18$ to $81.88 \pm$ 12.69 with a significant difference. And, the score of the volume of DTI (Unit, $\mathrm{mm}^{3}$ ) increased from $63.80 \pm 40.63$ to $120.80 \pm 115.16$. Conclusion the changes in the tract volume showed improvement in structure of the brain and upper limb function, which is resulted from the neuroplasticity. Therefore, mCIMT and APAs can improve Corticospinal tract (CST) through neuroplasticity and helpful in brain structure and upper limb function.
\end{abstract}

Key words APAs, CIMT, Corticospinal Tract, MAL, Upper limb function

Corresponding author Young-Dong Kim (exptkyd@gmail.com)

Received date 07 Jan 2020

Revised date 10 Feb 2020

Accept date 13 Feb 2020

\section{Introduction}

Patients make the action slow and discordant after stroke and tend to avoid movements that they used to do. Appropriate movements based on sensory information and feedback can be diminished by hindered sensory deficit. Perception, tactile discrimination and passive movements can be difficult to make after losing proprioception and tactile sensation. ${ }^{1)}$ Beside, stroke patients with the loss of proprioception of the hand loose an ability to get sensation of body parts and affect stability, perception of objects detected by the hand, hand motion control and motor function. ${ }^{2)}$ Without the hand function such as movement and sensation, it is difficult to distinguish the shape, material, size and temperature of an object. ${ }^{3)}$ So, movements need to get the receptors activated and feel sensation. Sensation depends on motor activities and

http:dx.doi.org/10.17817/2020.02.10.111530 vice versa because sensation and motor activities are closely interconnected with each other. ${ }^{4}$ Stroke patients reported that traditional rehabilitation therapy for upper limb is not shown much benefit than the lower limb. ${ }^{5)}$ It can be one of the reasons that walking needs a small recovery but the upper limb function involves diverse activities like reaching, grasping, holding and coordinating. As the hemiplegic time goes on, the use of the affected upper limb tremendously decreases and gets worsen. As well, the dominant use of the less affected upper limb in fulfilling daily activities could have a negative effect upon the functional recovery of the affected upper limb. ${ }^{\text {) }}$ Hemiplegic patients benefit from constraint induced movement therapy (CIMT) on the usage and function of the affected upper limb ${ }^{7)}$ or modified CIMT increases these in subjects with acute, subacute and chronic stages. ${ }^{8)}$ The previous study revealed the consequences of CIMT in the function of behavior and the brain, and ways to benefit from CIMT in hemi- 
plegic patients.9) All the methods excepting diffusion-tensor imaging (DTI) have been used to verify the mechanism of the neural system which support the clinical value of CIMT. ${ }^{10)}$ And, the corticospinal tract (CST) is the most important structure for motor control, especially for fine motor control of the hand in humans. ${ }^{11)}$ Neuroplasticity refers to the ability of modifying and reorganizing neurons and brain in structure and function by chaining the strength of synapses, dendrites, axons and the synaptic vesicular pool. ${ }^{12)}$ In addition, neuroplasticity is one of the consequences of improved motor system through CIMT. ${ }^{13)}$ In human movement, feed-forward and feedback control are anticipated and followed in initiation and ongoing movements, respectively. ${ }^{14)}$ Anticipatory postural adjustments (APTs) is an important component for postural control. ${ }^{15)}$ Therefore, this study aimed to verify whether modified CIMT (mCIMT) combined with APTs leads to improved corticospinal tract in hemiplegic patients.

\section{Materials and methods}

Nine hemiplegic patients after stroke from Daejeon Medical Care Hospital were participated in this study. The subjects were informed the purpose and methods before participating in this study. And, the subjects handed in the informed consent in advance. The inclusion criteria were as follows: 1) more than two years stroke, 2) diagnosed stroke by brain Computed tomography or Magnetic resonance imaging, 3) no more than $20^{\circ}$ wrist flexion, 4) no more than $10^{\circ}$ finger extension ${ }^{16)}$, 5) no seizures within the last 6 months, and 6) ability to understand the instruction.

A shaping which consists of Modified CIMT (mCIMT) and APAs was performed for 30 minutes per day, 5 days a week, for 4 weeks, without the less affected arm restricted from June $25^{\text {th }}$ to July $24^{\text {th }}, 2016$. And, the subjects were tested with Motor Activity Log (MAL) before and after the intervention. However, three out of 8 subjects did not get DTI clear enough to analyze so that they were excluded in the result of the CST tract volume.

\section{The shaping procedure was as follows;}

1) choosing tasks customized to address the motor deficits that individual patient had, 2) if the subjects were not able to complete a movement at first, having them help to perform the movement, and 3) giving positive verbal feedback for progress in task challenge according to the patients' improvement. ${ }^{17)}$

\section{Motor Activity Log (MAL)}

MAL is measured how patients use each limb by a semi-structured interview and independently rate how much and how well the patients have used the affected arm in 30 different activities of daily livings in the past week. Patients and care givers use amount of use (AOU) scale which is a 6-point to rate how much they are using the affected arm, and quality of movement (QOM) scale which is a 6-point to rate how

Table 1. General characteristics of the subjects.

\begin{tabular}{cccl}
\hline Patient & Age/Sex & Injury type & affected side \\
1 & $59 / \mathrm{F}$ & Rt. BG infarction & Lt. \\
2 & $56 / \mathrm{M}$ & Rt. MCA infarction & Lt. \\
3 & $33 / \mathrm{M}$ & Lt. ICH & Rt. \\
4 & $54 / \mathrm{M}$ & Lt. ICH & Rt. \\
5 & $24 / \mathrm{F}$ & Lt. Traumatic SDH & Rt. \\
6 & $64 / \mathrm{M}$ & Rt. Pontine infarction & Lt. \\
7 & $65 / \mathrm{F}$ & Rt. Pontine infarction & Lt. \\
8 & $41 / \mathrm{F}$ & Rt. BG ICH & Lt. \\
\hline
\end{tabular}

F, Female; M, Male; BG, Basal Ganglia, MCA, Middle Cerebral Artery; ICH, Intra-Cerebral Hemorrhage; SDH, Subdural Hemorrhage; Rt, Right; Lt, Left 
well they are using it. ${ }^{17)}$

\section{Diffusion Tensor Imaging (DTI)}

Diffusion tensor imaging which is a three-dimensional visualized version can visualize the integrity and architecture of white-matter tracts such as the corticospinal tract. $^{18)}$

Tract volume (TV)

Tract volume is measured by counting the number of voxels contained within a tract, and thus, reflect the total number of fibers in the tract. ${ }^{19)}$

SPSS for windows 12.0 was used for statistical analysis. Wilcoxon Signed Rank Test was used for hand function and CST volume before and after the mCIMT+APAs. A $\alpha=.05$ level of significance was used for the statistical test.

\section{RESULTS}

The MAL AOU increased from $32.00 \pm 32.04$ to 65.25 \pm 23.63 with a significant difference. The MAL QOM increased from $55.63 \pm 21.18$ to $81.88 \pm 12.69$ with a significant difference. (Table 2)

The score of the volume of DTI increased from $63.80 \pm 40.63$ to $120.80 \pm 115.16$. (Table 3 )

The volume of the corticospinal tract increased in most subjects.

\section{DISCUSSION}

Most of the daily activities after stroke are influenced by the impaired upper limb function. ${ }^{20)}$ CIMT based on neuroplasticity restrains the less affected arm and gets the affected upper limb moved as much as possible, which can help hemiplegic patients after stroke get over the learned nonuse. It also makes the arm function more efficient permanently resulted from the cerebral motor area extended in the brain which is in charge of upper limb function. Or, it unmasks a new motor area by doing repetition of functional exercise with the affected arm. $^{21)}$ In the previous study re-

Table 2. Variations of MAL.

$(\mathrm{N}=8)$

\begin{tabular}{ccccc} 
& Before & After & $z$ & $p$ \\
MAL AOU & $32.00 \pm 32.04$ & $65.25 \pm 23.63$ & -2.52 & $0.01^{*}$ \\
MAL QOM & $55.63 \pm 21.18$ & $81.88 \pm 12.69$ & -2.52 & $0.01^{*}$ \\
\hline
\end{tabular}

Unit, score; $\mathrm{M} \pm \mathrm{SD}$, Mean \pm standard deviation; * $\mathrm{p}<.05$; MAL, Motor Activity Log; AOU, Activity of use, QOM: Quality of movement

Table 3. Variation of the tract volume of DTI.

\begin{tabular}{cccccc} 
& Before & After & $z$ & $p$ \\
CST & $63.80 \pm 40.63$ & $120.80 \pm 115.16$ & -1.21 & 0.22 \\
\hline Unit,
\end{tabular}

Unit, $\mathrm{mm}^{3} ; \mathrm{M} \pm \mathrm{SD}$, Mean \pm standard deviation; DTI, diffusion tensor imaging; CST, Corticospinal tract

Table 4. Variations of the volume of the corticospinal tract.

\begin{tabular}{|c|c|c|c|c|}
\hline & \multicolumn{2}{|c|}{ Affected } & \multicolumn{2}{|c|}{ Unaffected } \\
\hline & $\begin{array}{c}\text { Before } \\
\text { Tv }\end{array}$ & $\begin{array}{l}\text { After } \\
\text { Tv }\end{array}$ & $\begin{array}{c}\text { Before } \\
\text { Tv }\end{array}$ & $\begin{array}{l}\text { After } \\
\text { Tv }\end{array}$ \\
\hline Patient 1 & 88 & 11 & 687 & 799 \\
\hline Patient 2 & 10 & 21 & 389 & 316 \\
\hline Patient 3 & 113 & 254 & 712 & 828 \\
\hline Patient 4 & 70 & 231 & 773 & 1246 \\
\hline Patient 5 & 38 & 87 & 858 & 814 \\
\hline
\end{tabular}




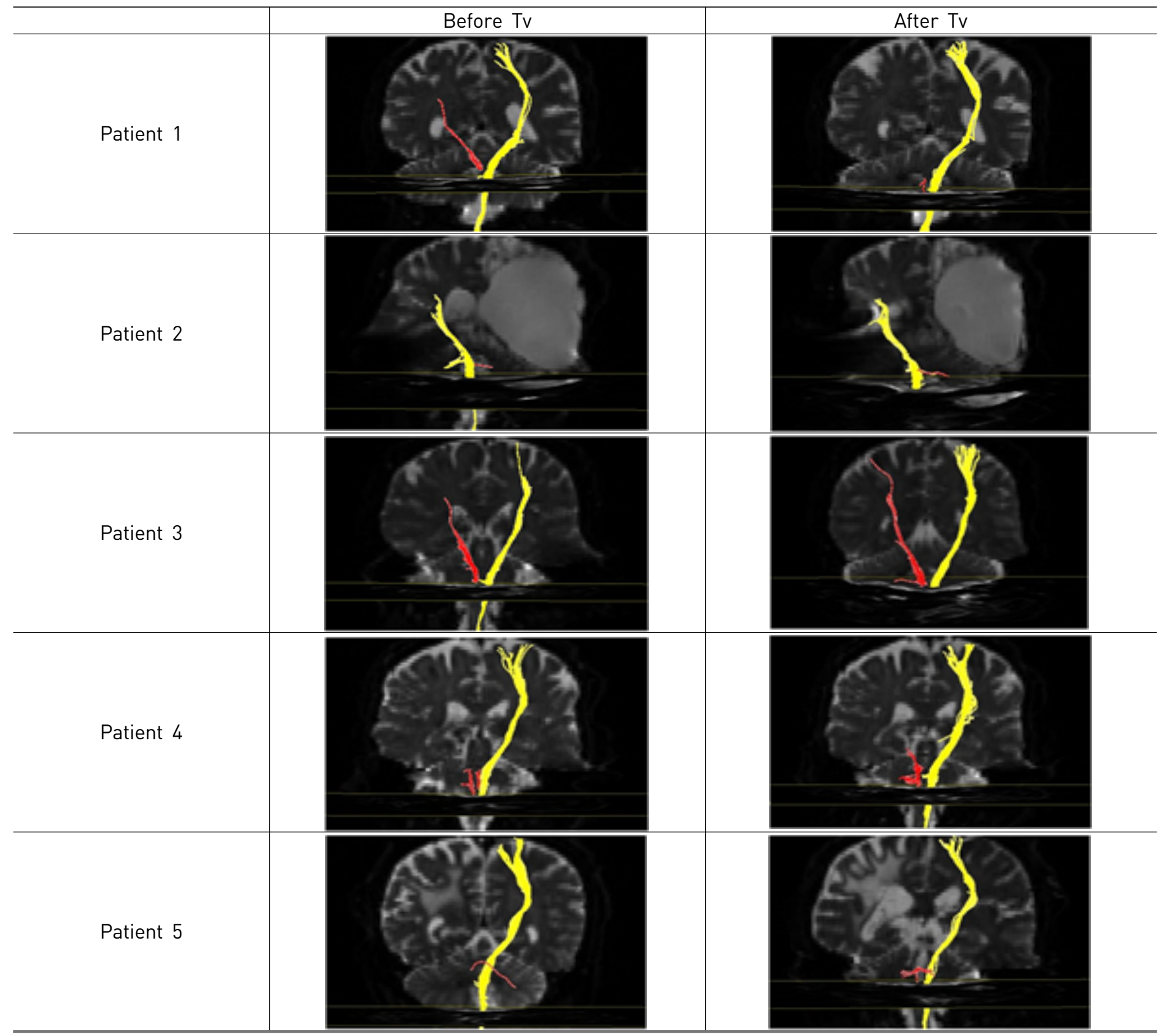

Tv: Tract volume

Figure 1. The volume of the corticospinal tract.

ported that CIMT was applied to fifteen hemiplegic patients and the patients were not allowed to move the less affected arm. And, the patients did exercises with the affected arm for seven hours a day for eight days long, which ended up with an outstanding improvement after all. ${ }^{22)}$ Preparatory APAs (pAPAs) which is occurring before voluntary limb movement keeps posture in stable by adapting to any perturbation. ${ }^{23)}$ The selected motor program should go with APAs in the trunk prior to reaching for an object with the upper limb. Otherwise, the patients might use a different strategy. ${ }^{24)}$ The previous study reported that MAL had statistically significant difference after applying CIMT to the subjects. ${ }^{25)}$ And, the previous study showed that the subjects who were applied CIMT got to have significant differences in MAL AOU and QOM. ${ }^{8)}$ In this study, both MAL AOU and QOM had statistically significant differences. As a result, CIMT can be a rehabilitation approach for hemiplegic patients with mild to moderately severe upper limb 
motor deficiency. The previous study revealed that transcranial magnetic stimulation (TMS) increased the number of scalp locations that made motor-evoked potentials in the affected hand. There was the ipsilesional motor map which was smaller at baseline and enlarged after CIMT and vice versa in the contralesional motor map. In this way, CIMT seemed to rebalance representations in the hand motor areas in the brain hemispheres. ${ }^{26)}$ The previous study showed that improved motor function after CIMT improved activity in the peri-infarct zone and both hemispheres. ${ }^{27)}$ In the previous study, all the methods (TMS, functional Magnetic Resonance Imaging (fMRI), structural MRI) except DTI have been used to verify the neural mechanisms in order to support the clinical effects of CIMT. ${ }^{10)}$ In this study, DTI was used to enable to numerically evaluate the state or change in a neural tract and motor recovery mechanisms in patients with hemiplegia. In the previous study, evaluation of the general state, changes in neural tracts, injury severity of a lesion or lesion site was conducted and estimation of changes in the neural tracts was followed by. On the top of that, the changes of the nucleus in the brain were evaluated. ${ }^{28)}$ In this study, even though DTI of 3 out of 8 subjects were not driven due to lack of clarity, DTI before and after mCIMT was used to verify the changes in the volume of CST of the affected hemisphere in the brain and motor function. As a result, the changes in the tract volume showed improvement in structure of the brain and upper limb function, which is resulted from the neuroplasticity. Therefore, mCIMT and APAs can improve CST through neuroplasticity and helpful in brain structure and upper limb function.

\section{References}

1. Mauguière F, Desmedt JE, Courjon J. Astereognosis and dissociated loss of frontal or parietal components of somatosensory evoked potentials in hemispheric lesions: Detailed correlations with clinical signs and computerized tomographic scanning. Brain. 1983;106(2):271-311.

2. Jeannerod M. The neural and behavioural organization of goal-directed movements. Clarendon Press/Oxford University Press, 1988.
3. Hogan N, Winters JM. Principles underlying movement organization: Upper limb. In: Multiple muscle systems. Springer, 1990;182-94.

4. Brodal P. The central nervous system: Structure and function. Oxford University Press, 2004.

5. Basmajian JV. The winter of our discontent: Breaking intolerable time locks for stroke survivors. Arch Phys Med Rehabil. 1989;70(2):92-4.

6. Van der Lee JH, Wagenaar RC, Lankhorst GJ, et al. Forced use of the upper extremity in chronic stroke patients: Results from a single-blind randomized clinical trial. Stroke. 1999;30(11):2369-75.

7. Duncan PW. Synthesis of intervention trials to improve motor recovery following stroke. Top Stroke Rehabil. 1997;3(4):1-20.

8. Page SJ, Levine $\mathrm{P}$, Leonard $\mathrm{A}$, et al. Modified constraint-induced therapy in chronic stroke: Results of a single-blinded randomized controlled trial. Phys Ther. 2008;88(3):333-40.

9. Tarkka IM, Könönen M. Methods to improve constraint-induced movement therapy. NeuroRehabil. 2009;25(1):59-68.

10. Wittenberg GF, Schaechter JD. The neural basis of constraint-induced movement therapy. Curr Opin Neurol. 2009;22(6):582-8

11. CANEDO A. Primary motor cortex influences on the descending and ascending systems. Prog neurobiol. 1997;51(3):287-335.

12. Kleim JA, Barbay S, Nudo RJ. Functional reorganization of the rat motor cortex following motor skill learning. J neurophysiol. 1998;80(6):3321-5.

13. Gauthier LV, Taub E, Perkins C, et al. Remodeling the brain plastic structural brain changes produced by different motor therapies after stroke. Stroke. 2008;39(5):1520

14. Schepens B, Drew T. Independent and convergent signals from the pontomedullary reticular formation contribute to the control of posture and movement during reaching in the cat. J neurophysiol. 2004;92(4):2217-38.

15. Massion J. Movement, posture and equilibrium: Interaction and coordination. Prog neurobiol. 1992;38(1):35-56.

16. De Souza L, Hewer RL, Lynn P, et al. Assessment of recovery of arm control in hemiplegic stroke patients. 2. Comparison of arm function tests and pursuit tracking 
in relation to clinical recovery. Int rehabil med. 1980;2(1):10-6.

17. Taub E, Uswatte G, Pidikiti R. Constraint-induced movement therapy: A new family of techniques with broad application to physical rehabilitation-a clinical review. J Rehabil Res Dev. 1999;36(3):237-51.

18. Mori S, Crain BJ, Chacko VP, et al. Three-dimensional tracking of axonal projections in the brain by magnetic resonance imaging. Ann Neurol. 1999;45(2):265-9.

19. Pagani E, Agosta F, Rocca MA, et al. Voxel-based analysis derived from fractional anisotropy images of white matter volume changes with aging. Neuroimage. 2008;41(3): 657-67.

20. Page SJ, Levine P, Sisto S, et al. Stroke patients' and therapists' opinions of constraint-induced movement therapy. Clin Rehabil. 2002;16(1):55-60.

21. Koop CE, Juberg DR, Benedek EP, et al. A scientific evaluation of health effects of two plasticizers used in medical devices and toys: A report from the american council on science and health. MedGenMed. 1999;1(1).

22. Miltner WH, Bauder H, Sommer M, et al. Effects of constraint-induced movement therapy on patients with chronic motor deficits after stroke: A replication. Stroke. 1999;30(3):586-92.
23. Horak FB. Postural orientation and equilibrium: What do we need to know about neural control of balance to prevent falls? Age ageing. 2006;35(suppl_2):ii7-ii11.

24. Sue R, Meadows Linzi L, Concept B. Theory and clinical practice in neurological rehabilitation. Charlottesville, VA: Wiley-Blackwell/John Wiley \& Sons; 2009.

25. Kim C. The application of constraint-induced movement therapy (cimt) on hemiplegia. Inje university. Dissertation of Master's Degree, 2002.

26. Liepert J, Miltner W, Bauder H, et al. Motor cortex plasticity during constraint-induced movement therapy in stroke patients. Neurosci Lett. 1998;250(1):5-8.

27. Levy CE, Nichols DS, Schmalbrock PM, et al. Functional mri evidence of cortical reorganization in upper-limb stroke hemiplegia treated with constraint-induced movement therapy. Am J Phys Med Rehabil. 2001;80(1):4-12.

28. Little D, Kraus M, Joseph J, et al. Thalamic integrity underlies executive dysfunction in traumatic brain injury. Neurology. 2010;74(7):558-64. 